\title{
Generalization of the Jared and Ennis method of complex transmittance objects for the generation of synthetic discriminant function filters
}

\author{
Encarnación Pleguezuelos, Ignasi Labastida, Mario Montes-Usategui, \\ Santiago Vallmitjana, and Artur Carnicer
}

\begin{abstract}
We present a simple method of constructing synthetic discriminant function filters optimized to take into account the modulation of liquid-crystal devices. This relaxation algorithm, a generalization of the Jared and Ennis method, is an iterative method that includes arbitrary modulations for both scene and filter, extending the problem to the complex plane. Simulated and experimental results obtained in a VanderLugt correlator are presented for a two-class recognition problem. The optimal number of images needed to describe an object in a filter generated in this way is discussed, and the influence of the spatial light modulation resolution on the correlation is studied. (C) 2004 Optical Society of America

OCIS codes: $\quad 070.4550,070.6110,100.6740,230.3720$.
\end{abstract}

\section{Introduction}

Filter design is a highly dynamic field in optical pattern recognition research. If general distortion invariance is desired, one of the most common filters used is the synthetic discriminant function (SDF) filter. ${ }^{1}$ This type of filter is constructed as a function of a number of representative views of an object with a correlation response fixed for all of them. Depending on the images chosen, we would be able to construct a filter that is invariant to the type of distortion required. Other filters are invariant to a particular distortion, such as circular harmonic filters, ${ }^{2}$ which achieve the same correlation response for any inplane rotation of the object with a single term of the harmonic decomposition of an image for the correlation, or radial harmonic filters, ${ }^{3}$ which do the same for scale variations. The main drawback of these filters is that a single harmonic contains only partial information about the object. Another example for rotation invariance in optical pattern recognition is based on a different approach: time multiplexing, ${ }^{4}$

The authors are with the Departament de Física Aplicada i Òptica, Universitat de Barcelona, Avinguda Diagonal 647, Barcelona E08028, Spain. The e-mail address for E. Pleguezuelos is encarni@fao.ub.es.

Received 17 February 2004; revised manuscript received 7 July 2004; accepted 15 July 2004.

0003-6935/04/305647-08\$15.00/0

(C) 2004 Optical Society of America which consists in the mechanical rotation of the reference while the correlation is captured.

An algorithm for the generation of correlation filters has to take into account the characteristics of the spatial light modulator (SLM) where the images will be displayed. Most SLMs can only accommodate a small fraction of the complex plane, so the filter has to be adapted to the possible values during its construction. A single-image filter that includes these considerations is the minimum Euclidean distance optimal filter (MEDOF). ${ }^{5}$ This type of filter optimizes different metrics in its design, such as the signal-to-noise ratio in the correlation plane or the peak sharpness. Previous attempts have been made to include adaptation to display devices in the SDF by use of the phase of a composite filter generated in the conventional way. ${ }^{6}$ The results were not appropriate because the SDF constraints were no longer met after the adaptation process, as Casasent and Rozzi have shown. ${ }^{7}$ Since then, however, other algorithms have optimized the SDF to phase-only modulation. These include Kallman's method, ${ }^{8}$ which is computationally intensive, or the Jared and Ennis method, ${ }^{9}$ which is an iterative procedure and which comprises the starting point from the method presented in the present paper. Montes-Usategui et $a l .{ }^{10}$ have presented an algorithm that achieves the generation of the SDF, taking into account an arbitrary modulation for the filter. Nevertheless, none of these authors mentions the inclusion of scene modulation due to the displaying device. Juday ${ }^{11}$ ob- 
tained a solution that optimizes metrics of several kinds, such as the Fisher ratio or the Bayes error, including the constraints imposed by the limited modulation, with the same philosophy as in the MEDOF filters ${ }^{5}$ but extending it to account for a set of images, although he does not give a practical algorithm for computing the filters. Our aim is to include the modulation of both SLMs in the filter's design to implement it experimentally, generalizing the Jared and Ennis method to the complex plane, to allow arbitrary modulations.

This paper is organized as follows. In Section 2 we review the Jared and Ennis method. In Section 3 we explain the algorithm used to generalize this method to complex-valued scenes and arbitrary modulations. In Section 4 some simulated results are presented, and we discuss the optimal conditions to this type of filter construction, analyzing the optimal number of images that a filter should contain and the influence of the number of gray levels available due to the modulation. In Section 5, some experimental results are shown, and the paper is closed by the conclusions in Section 6.

\section{Jared and Ennis Method}

The Jared and Ennis method is an iterative algorithm for the construction of phase-only SDF filters that, since its publication, has been widely used, ${ }^{12,13}$ possibly because of its mathematical simplicity. This fact, joined to its fast convergence on the solution, is the main advantage that made us consider this method as the starting point in the development of our algorithm. As we have already commented, SDF filters are one of the possible choices to achieve distortion invariance. The reason is that different views of an object are considered in the construction of a single filter, thus assuming that intermediate cases should not be rejected because of the criteria that we impose on the output correlation.

In SDF filters, the correlations between each training image and the reference image are imposed, and we calculated the filter considering these constraints as follows:

$$
\left[t_{n}(x, y) \otimes h(x, y)\right]_{(0,0)}=c_{n},
$$

where $t_{n}(x, y)$ is the $n$ image of the training set and $h(x, y)$ is the filter in the real space. $c_{n}$ is the central correlation amplitude, which is fixed to the desired value; for example, it can be 1 for the detected class and 0 for the rejected one. In the particular case of a filter being a linear combination of images, the result is a system of linear equations ( $N$ equations, one for each image of the training set) that can be analytically solved. If an arbitrary modulation is considered, then the system has no analytical solution in general.

The Jared and Ennis method first considers the filter generated as a conventional SDF:

$$
h(x, y)=\sum_{n=0}^{N} a_{n} t_{n}(x, y),
$$

where $a_{n}$ is the coefficient of the $t_{n}(x, y)$ image. The images of the training set are not adapted to any modulation, and for this reason they are considered real-valued images. Whereas in the original method the $a_{n}$ coefficients are considered real, in our implementation these parameters do not have this constraint. The filter, once constructed, is adapted to phase-only or binary phase-only modulation. The $N$ constraints in Eq. (1) are imposed, and the system of equations for the coefficients is solved by the following iteration:

$$
\begin{aligned}
a_{n}^{i+1} & =a_{n}^{i}+\beta\left(c_{n}-c_{0} \frac{m_{n}^{i}}{m_{0}^{i}}\right), \\
m_{n}{ }^{i} & =\left[t_{n}(x, y) \otimes h^{i}(x, y)\right]_{(0,0)},
\end{aligned}
$$

where $i$ is the iteration number and $\beta$ is a parameter that the convergence depends on. Jared and Ennis ${ }^{9}$ fixed this parameter to 0.3. $\quad m_{n}$ is the central correlation amplitude between each image of the training set and the filter generated with the coefficients found in the iteration $i$ once it has been adapted to the modulation. The initial conditions for the iteration are $a_{n}{ }^{0}=c_{n}$. When the $m_{n}{ }^{i}$ central correlation amplitudes are equal to the fixed ones $\left(c_{n}\right)$, the method has converged into the solution. Although the method presents advantages, there is a need for its generalization to any experimental filter modulation. Previous studies have appeared that are related to the generalization of the Jared and Ennis method, which have attempted to introduce more than one image in the generation of $\mathrm{MEDOFs}^{14}$ adapted to the filter modulation, without considering any scene modulation. The approach we present deals with these problems, as we show in Section 3.

\section{Generalization to the Complex Plane}

The method presented here takes into account modulations other than the ones applied in the original method, both for the filter and for the images considered in the filter's construction. These considerations are indispensable for the experimental verification of the method. The method developed can be used with any configuration, although we do not consider here the associated problem of which curves are more suitable for a given problem. ${ }^{15}$ In our case, the images will be displayed on two video graphics array liquid-crystal devices (LCDs) extracted from an Epson EMP-3000 video projector. From the different available curves, we chose to use a high-contrast configuration for the scenes and a phase-mostly configuration for the filter in this study. As already mentioned, they could be different configurations. The operative curves that represent these modulations are shown in Figs. 1(a) and 1(b), respectively. The adaptation of the filter to the phasemostly modulation is the result of our applying the minimum Euclidean distance of each complex value to the points represented in the curve, the only ones available in the operating configuration. We ob- 


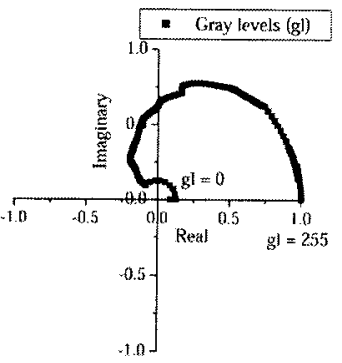

(a)

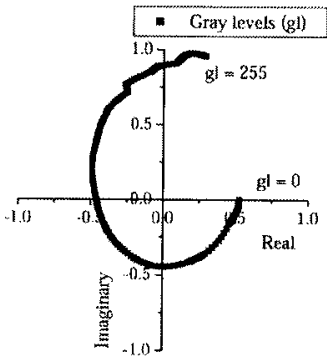

(b)
Fig. 1. Operative curves considered in the filter generation: (a) modulation of the scene (high-contrast configuration), (b) modulation of the filter (phase-mostly configuration).

tained both curves using an experimental characterization method. 16

The filter in the Fourier plane, $H(u, v)$, is generated as follows:

$$
H(u, v)=\mu_{f}\left(\mathscr{F}_{F}\left(\sum_{n=0}^{N-1} a_{n} M_{i}\left[t_{n}(x, y)\right]\right\}^{*}\right),
$$

where $\mathscr{F}_{F}$ indicates the Fourier transform. $M_{i}$ is the complex number that results when each gray level of the input image is displayed in the input SLM, and $\mu_{f}$ is the available value that has a minimum Euclidean distance to the value that the filter should have. To take into account the scale of the filter, we normalized its magnitude to 1.0 (the operative curve is normalized to the same value) and then collapsed its values to realizable ones by application of the minimum Euclidean distance. At this point, some kind of optimization could have been introduced instead of the simpler method we are using. Following the standard MEDOF practice, ${ }^{5}$ a multiplicative complex factor can be found that makes the scale of the filter and its absolute phase optimal with respect to the curve being used in terms of maximizing some kind of metric. The filter was generated as the linear combination of the training set once modulated, transformed into the Fourier plane, conjugated, and adapted at this point to the second modulation function, by means of the minimum Euclidean distance, as already stated above. Note that the images belonging to the training set are adapted to the curves in the object plane whereas the filter is adapted in the Fourier plane.

If the SDF conditions are imposed on the filter [Eq. (1)], the solution to the coefficients cannot be found analytically, and an iterative method has to be considered to solve the system of equations. In our method, the coefficients of the linear combination are not restricted to being real because phase is a desirable and necessary degree of freedom in the optimization, and so we allow them to have complex values so we can add parameters to the problem. Therefore the fixed correlations $c_{n}$ are also complex valued. We apply the formula of Eq. (3) of the Jared and Ennis method to iterate the real and imaginary parts

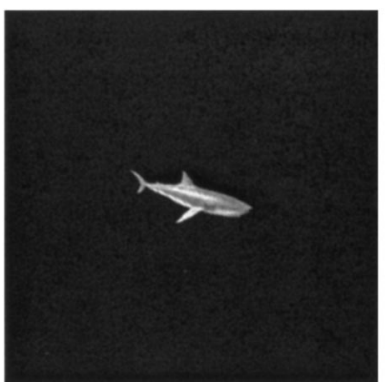

(a)

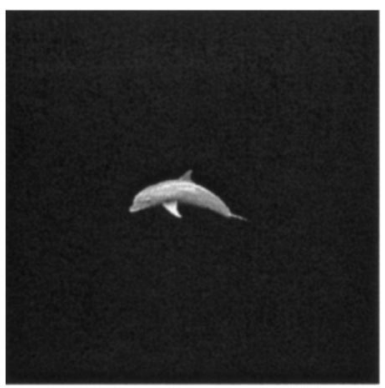

(b)
Fig. 2. Images of the training set: (a) class A, (b) class B.

of the coefficients separately, as shown in Eqs. (6) and (7):

$$
\begin{aligned}
& \mathscr{R}\left(a_{n}{ }^{i+1}\right)=\mathscr{R}\left(a_{n}{ }^{i}\right)+\beta\left[\mathscr{R}\left(c_{n}\right)-\mathscr{R}\left(c_{0}\right) \frac{\mathscr{R}\left(m_{n}{ }^{i}\right)}{\mathscr{R}\left(m_{0}{ }^{i}\right)}\right], \\
& \mathscr{I}\left(a_{n}{ }^{i+1}\right)=\mathscr{I}\left(a_{n}{ }^{i}\right)+\beta\left[\mathscr{I}\left(c_{n}\right)-\mathscr{I}\left(c_{0}\right) \frac{\mathscr{I}\left(m_{n}{ }^{i}\right)}{\mathscr{I}\left(m_{0}{ }^{i}\right)}\right] .
\end{aligned}
$$

Parameter $\beta$ was fixed to 0.3 as in the original method in both Eq. (6) and (7) to achieve the simultaneous convergence of the real and imaginary parts of the coefficient. $m_{n}{ }^{i}$ has the same definition as in Eq. (4) but the filter and the training set image were adapted to its correspondent modulation, as in Eq. (5). The initial coefficients were chosen to be equal to the $N$ central correlations $c_{n}$, which were considered equal to $[(1,1) / \sqrt{2}]$, thus fixing the central correlation amplitude to 1 for images of the training set that belong to a detected class. These values are $c_{n}=(0,0)$ for a rejected class. As the variable that we want to set is the central correlation amplitude and not its phase, we have a degree of freedom that could be used to optimize other metrics in addition to the correlation amplitude, as others have done, ${ }^{11}$ but we do not exploit this freedom in the present study as the conditions mentioned above are enough to achieve convergence of the algorithm, which is our basic goal. As the algorithm fixes only the central correlation value, the maximum for a correlation with a rejected image could be placed in another point of the correlation plane. This fact can lead to false detections, which is the SDF filters' main drawback.

\section{Simulated Results}

The training set used in this study is compounded by in-plane rotations of the images shown in Fig. 2, where a shark and its rotations form class A and a dolphin and its rotations form class B. Any other distortion could be considered, not just a simple rotation. To check the stability of the method, we generated a filter from four images, two belonging to each class, detecting class A with the modulations shown in Figs. 1(a) and 1(b). We generated the rest of the filters discussed in this paper considering the same modulations for the filter and the scenes. Figure 3 shows the evolution of the central correlation ampli- 


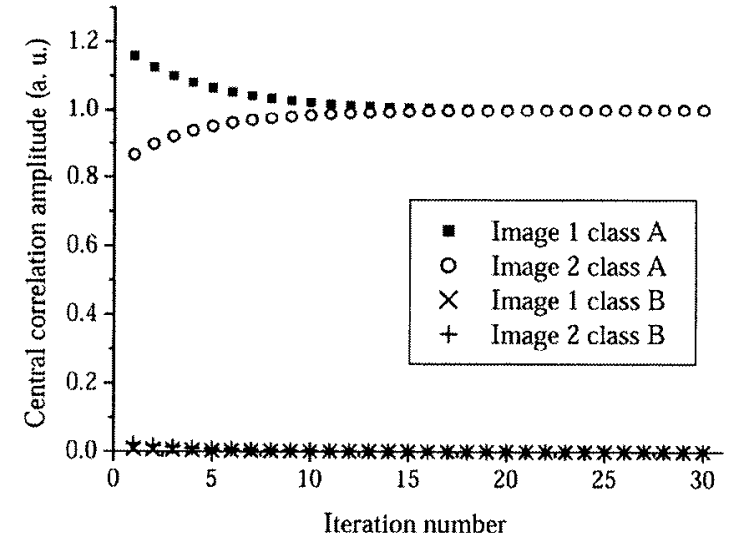

Fig. 3. Evolution of the central correlation amplitude along the iteration in the generation with the generalized method of a filter that includes four images, two of each class, with the detection of class A.

tude along the iteration. The method converges after a few iterations to the fixed values. The time per iteration is approximately $1 \mathrm{~min}$ in a $1.2-\mathrm{GHz}$ Pentium IV processor during the generation of a filter from 36 images with the size of $512 \times 512$ pixels, and is thus not a critical issue. The method is stable and maintains the mathematical simplicity of the original one.

We carried out some simulations to test the performance of the filters generated with the method presented in this paper using the operative curves shown in Fig. 1. First, we wanted to see if the filter detects all the images in the training set with the same correlation peak intensity. To verify this, we constructed a filter containing 36 images belonging to class A (sharks with an in-plane rotation of $20^{\circ}$ each). Figure 4 shows the central correlation amplitude between each image of the training set and this filter. As can be seen, the values are equal for all the scenes.

To use the filters properly, the conditions in which the filter performs optimally must be established. In this paper we focus on three conditions: the number of images per filter, the separation in

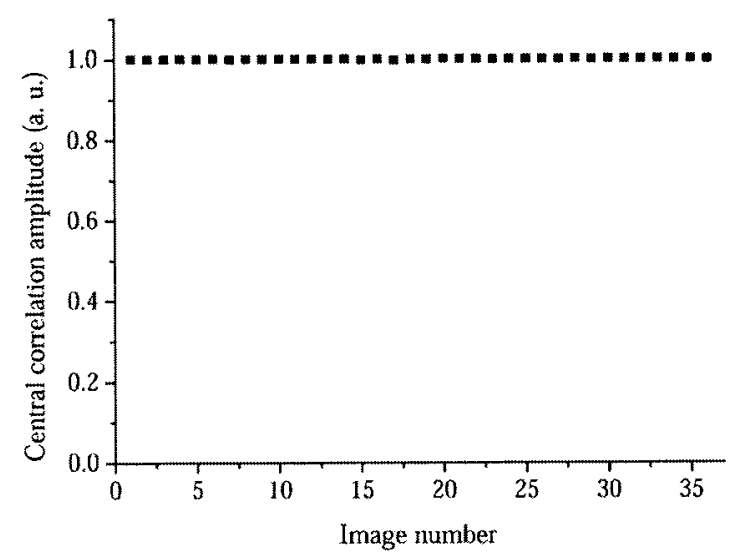

Fig. 4. Central correlation amplitude with each of the 36 images belonging to class A and a filter generated from all of them.

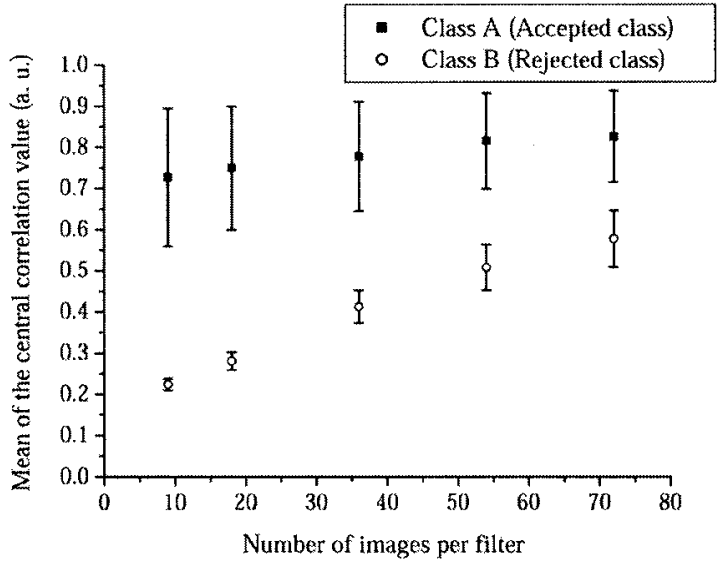

Fig. 5. Influence of the number of images per filter in the correlation. The filled squares show the mean value of the maxima of correlation between filters, which were generated from a number of images each rotated $5^{\circ}$, and images of the detected class, those belonging to the training set, and intermediate images. The open circles indicate the mean value of the maximum with images of the rejected class and thus should have a low value. The detected class is sharks, and the number of images in the filter is equal for each class. The correlation values are normalized to the autocorrelation, as in Figs. 4-9 and 11.

degrees between the rotations of the training set images, and the number of levels in which we codify the filter, in our case from 256 levels to binary filters.

First, there is a need to determine the number of images that a single filter should contain. The number has to be as large as possible, but there are other issues that we have to assume. The larger the number of images the filter contains, the noisier the correlation we would obtain. This parameter also influences the cross correlation. Considering a separation of $5^{\circ}$ between rotations and 256 gray levels in the LCD, we generated five filters containing a different number of images. Figure 5 shows the average of the maximum amplitudes of correlation for different filters, from a 9-image per class filter to a 72-image per class filter. The average of the correlation values for each filter was calculated with those images included in the filter and those that were not but corresponded to intermediate images and with an equal number of images of the rejected class for the mean value of class $B$. The error bars are the standard deviation of the correlations with the same images considered in the mean value. The values were normalized to the autocorrelation value. From the plot in Fig. 5 we can see that no more than approximately 20 images per filter should be included because the distance between the correlation with an object of the desired class and another object decreases. The threshold we have to impose on the correlation to determine whether detection has occurred is more difficult to set if the correlations between the target class and another class are similar. To quantify 
Table 1. Influence of the Number of Images per Filter in the Fisher Ratio

\begin{tabular}{cc}
\hline Number of Images per Class & Fisher Ratio \\
\hline 9 & 8.93 \\
18 & 9.56 \\
36 & 6.96 \\
54 & 5.67 \\
72 & 3.60 \\
\hline
\end{tabular}

the separability of each filter, we constructed the Fisher ratio, which is defined as

$$
J=\frac{\left(\langle I\rangle_{A}-\langle I\rangle_{B}\right)^{2}}{\sigma_{A}^{2}+\sigma_{B}^{2}}
$$

where $\langle I\rangle_{A}$ and $\langle I\rangle_{B}$ are the intensities averaged over images belonging to class $\mathrm{A}$ and $\mathrm{B}$, respectively, and $\sigma_{A}$ and $\sigma_{B}$ are the standard deviation values in the class. The mean and the standard deviation are calculated from the correlations with images that the filter has to detect, that is, images of the filter's training set and images with intermediate rotation degrees. One should expect a high Fisher ratio for a filter that is able to separate the two classes. In Table 1 we show the Fisher ratios corresponding to the filters in Fig. 5. The filter with a better Fisher ratio is the one constructed from 18 images.

Once this has been analyzed, the separability among training images is studied. This matter is highly dependent on the nature of the images themselves, as well as on the characteristics of the SLM. The correlation with rotations of the object that are not included in the training set will be larger if the angular separation between images of the training set is smaller. To find the optimal degree, we tested the performance of a single matched filter against the rotation of the input object, and the results are shown in Fig. 6. The maximum correlation amplitude with an image that is separated by $2^{\circ}$ from the image considered in the matched filter is below 0.5 of the

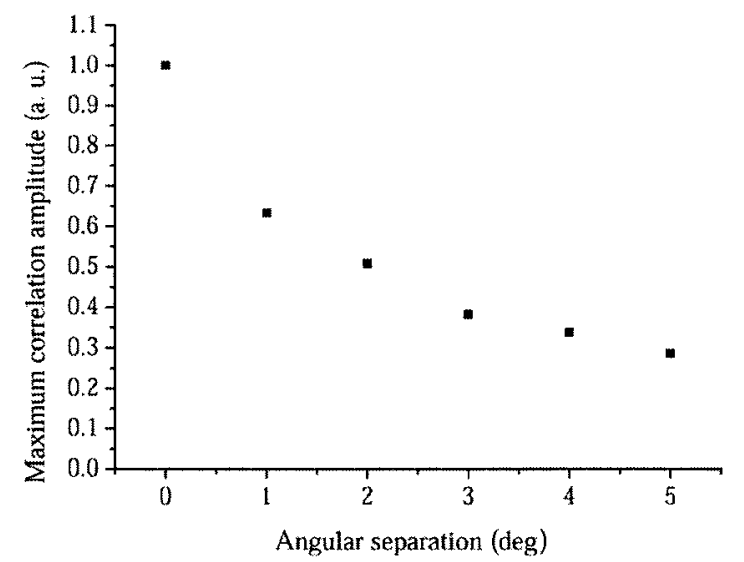

Fig. 6. Maximum correlation amplitude of a single matched filter against the rotation of the input object. At a rotation of $2^{\circ}$ the amplitude has decreased to half of the autocorrelation value.

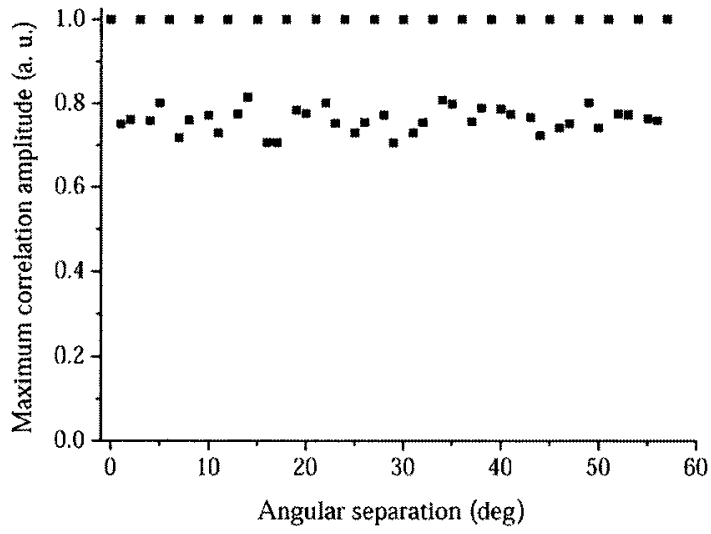

Fig. 7. Maximum correlation amplitude with a filter generated from 20 images, each rotated $3^{\circ}$ and both belonging to the training set and intermediate images.

autocorrelation maximum. If we consider a filter generated from 20 images rotated $3^{\circ}$ and test its performance against intermediate images, we can see that there is no image that results in a correlation below 0.7 of the correlation amplitude with the images in the training set (Fig. 7). Once this was verified, we constructed four filters containing 20 images of class A with a different sampling interval from 3 to $20^{\circ}$. In Fig. 8 we show the correlation mean values between these filters and, as in Fig. 5, with images belonging to class A (included or not in the filter) or with images of class B. The separation should not be more than $5^{\circ}$ because the separation between classes is too low to be accepted. It would be difficult to set a threshold value for an interval of $10^{\circ}$. In Table 2 we can see the Fisher ratios [Eq. (8)] for the filters used in Fig. 8. There is a great difference between the separability between classes in the case of $3^{\circ}$ and the other cases. If a sampling interval of $3^{\circ}$

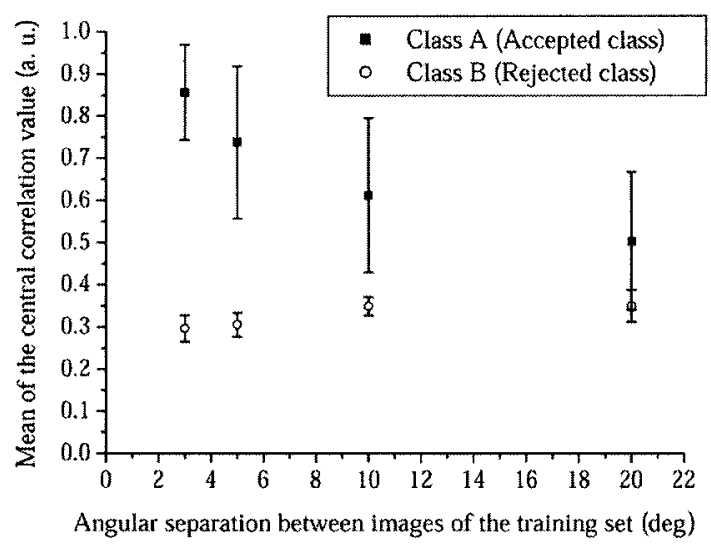

Fig. 8. Influence in the correlation of the sampling interval of the images in the training set. The filled squares show the mean value of the correlation maxima between filters and images of the detected class, both belonging to the training set and intermediate images. The open circles indicate the mean value of the maximum with images of the rejected class. The detected class is sharks. 
Table 2. Influence of the Angular Separation among Images in the Filter in the Fisher Ratio

\begin{tabular}{cc}
\hline Angular Separation & Fisher Ratio \\
\hline 3 & 22.76 \\
5 & 8.58 \\
10 & 2.04 \\
20 & 0.83 \\
\hline
\end{tabular}

is considered, we need six filters of 20 images to describe a full in-plane rotation of our object, which is a reasonable number.

Finally, we would like to study the influence of the LCD resolution in our filter. Taking a filter generated under the above conditions, that is, 20 images per filter and $3^{\circ}$ of rotation, we adapted it to different numbers of gray levels, from binary to 256 levels. The scene was codified into the same number of gray levels. Although we could leave scenes with the initial resolution, we consider that both SLMs are equal, and because of this, they should have the same resolution. The discretization was made in the following way: For the input we chose the levels that are as widely spaced as possible in the amplitude of the complex values available with the high-contrast configuration, and we do the same for the filter, but with the phase of the complex values in the phase-mostly configuration so as to cover the widest range of complex planes possible. Figure 9 shows the average of the maximum correlation values with filters adapted to modulations of $2^{n}$ levels. The scenes considered for the correlation in Fig. 9 are the same as in Figs. 5 and 8. The number of gray levels in the LCD turns out to be the parameter that has less influence on the correlation, at least for the scenes and the type of filter used in this study. We calculated the Fisher

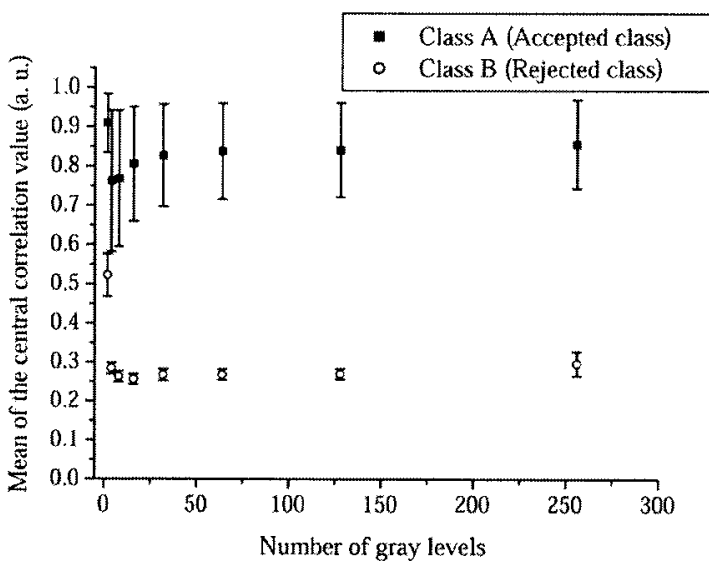

Fig. 9. Influence in the correlation of the number of gray levels in which the filter is adapted. The filled squares show the mean value of the maxima of correlation between filters, which were generated from 20 images of each class separated $3^{\circ}$, and images of the detected class, both belonging to the training set and intermediate images. The open circles indicate the mean value of the maximum with images of the rejected class. The detected class is sharks.
Table 3. Influence of the Number of Gray Levels in the Filter in the Fisher Ratio

\begin{tabular}{cc}
\hline Number of Gray Levels & Fisher Ratio \\
\hline 256 & 22.76 \\
128 & 22.38 \\
64 & 21.45 \\
32 & 18.20 \\
16 & 14.07 \\
8 & 8.43 \\
4 & 7.07 \\
2 & 17.48 \\
\hline
\end{tabular}

ratio for these filters and they are shown in Table 3. We can see that the Fisher ratio decreases with the number of gray levels, except for the binary case, but this is due to the fact that the standard deviation is lower in this case than in the others.

The results led us to conclude that in the studied cases the optimal conditions for the filter generation are $3^{\circ}$ of angular separation between images of the training set, each filter containing 20 images, and with a codification of a minimum of 32 levels, although we have shown that the number of levels is not an important parameter in our problem. We could set a threshold for the normalized maximum correlation amplitude of approximately 0.65 for the object to be detected, considering that, in the simulations we carried out with a filter in these conditions, we obtained a minimum of approximately 0.70 . In Section 5 we present some experimental results to check the performance of the filters.

\section{Experimental Results}

The experimental setup used in this paper is a VanderLugt convergent correlator (Fig. 10) with two video graphic array LCDs removed from an Epson EMP-3000 video projector. We used a $\mathrm{He}-\mathrm{Ne}$ laser source of $632.8 \mathrm{~nm}$ and a CCD camera to capture the correlation plane. The LCDs work under the configurations shown in Fig. 1. As the filters generated with our method are optimized to the configurations of both LCDs, we can check the performance of the algorithm experimentally.

A filter containing 20 images belonging to class A (sharks) each separated $3^{\circ}$ adapted to 32 gray levels was constructed. This filter has to detect sharks

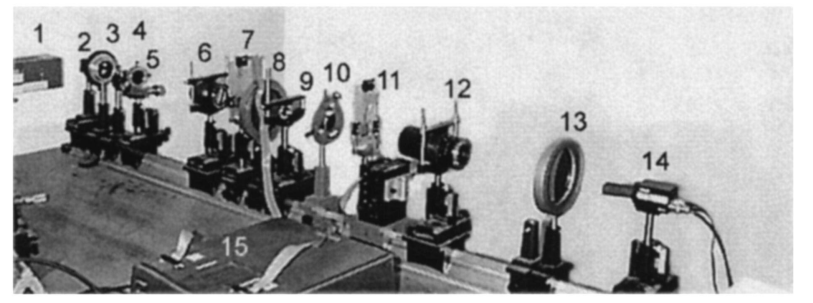

Fig. 10. Picture of the VanderLugt convergent correlator. 1, laser; 2 , mirror; $3,8,10$, and 13 , polarizing elements; 4 , attenuation filter; 5 , pinhole; 6 and 12, convergent lenses; 7 and 11, LCD; 9 , divergent lens; 14, CCD camera; 15, video projector. 


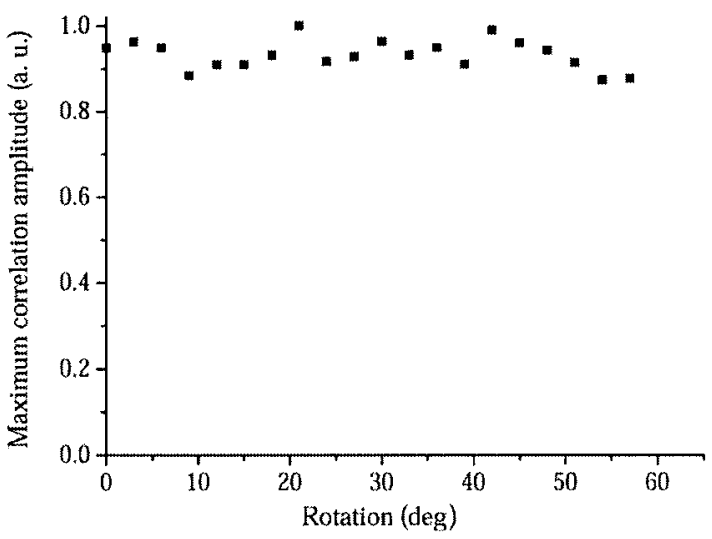

Fig. 11. Maximum correlation amplitude captured experimentally with each of the 20 images included in the training set; the sharks had a rotation of $3^{\circ}$ each.

with a rotation of $0^{\circ}-57^{\circ}$. We captured the correlation planes obtained when this filter was displayed on the second modulator, and the first modulation had scenes containing each shark of the training set separately. Figure 11 shows the maximum correlation amplitude, which was normalized to the maximum of all values and presents a mean value of 0.93 with a standard deviation of 0.03 . The minimum value is 0.87 , so we can conclude that the filter detects all the sharks, as expected. The main error sources may be misalignments of the correlator and the stability of the operative curves. ${ }^{17}$

Figures 12 and 13 show correlation planes captured experimentally and the scenes displayed on the first LCD. The rotation degrees are indicated in the scenes, and the correlation intensity maxima are normalized to the maximum of the image. In Fig. 12 we can see that there is detection for an image belonging to the training set and for an intermediate degree, with almost the same intensity. The correlation intensity is lower for a dolphin, which is not the target and so has to be rejected. Figure 13 shows the correlation with a scene that contains an object of the training set and one dolphin. Clearly, there is no detection for the dolphin in this case because the correlation maximum 0.29 is lower than the fixed

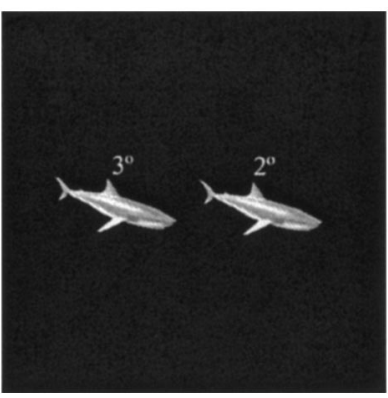

(a)

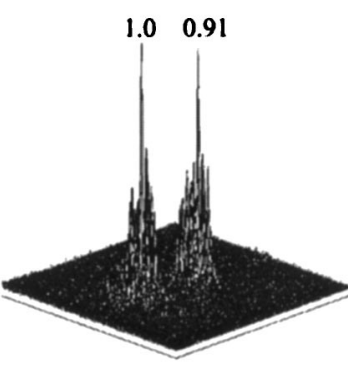

(b)
Fig. 12. (a) Input scene with two images of class A and (b) experimental correlation plane captured with a filter generated from 20 images belonging to class $\mathrm{A}$.

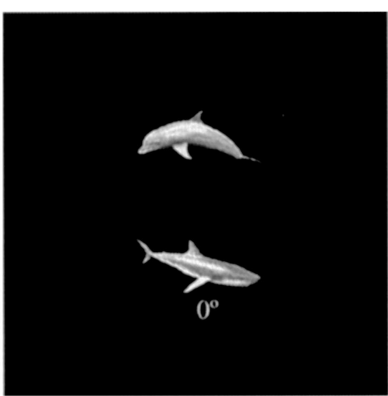

(a)

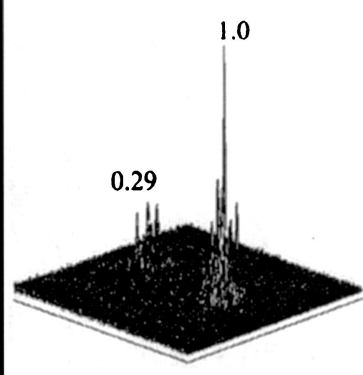

(b)
Fig. 13. (a) Input scene with an image of class A and another of class B and (b) experimental correlation plane captured with a filter generated from 20 images belonging to class A.

threshold 0.65. To summarize, the experimental results are in agreement with the simulated ones, and the correct behavior of the algorithm has been proved.

\section{Conclusions}

We propose an algorithm for the design of SDF filters adapted to arbitrarily constrained devices to display images in a VanderLugt correlator, taking into account the device modulation for scenes and filter. The algorithm is a generalization of the Jared and Ennis method for complex-valued images, which maintains its simplicity and its fast convergence on the solution. The simulated results allowed us to find the optimal conditions of performance for these filters, analyzing issues such as the number of images per filter, the LCD resolution, and the angular separation of the images in the training set for the considered distortion, the in-plane rotation. Any other distortion could have been chosen by use of other images in the training set. Experimental results obtained in a convergent VanderLugt correlator are shown and present good agreement with the simulated results.

This research has been partially funded by the Comisión Interministerial de Ciencia y Tecnologia through project DPI 2001-3365 and by the Comissió Interdepertamental de Recerca i Tecnologia through project 2001SGR00079.

\section{References}

1. C. F. Hester and D. Casasent, "Multivariant technique for multiclass pattern recognition," Appl. Opt. 19, 1758-1761 (1980).

2. Y. N. Hsu and H. H. Arsenault, "Optical character recognition using circular harmonic expansion,” Appl. Opt. 21, 4016-4019 (1982).

3. D. Mendlovic, E. Marom, and N. Konforti, "Shift and scale invariant pattern recognition using Mellin radial harmonics," Opt. Commun. 67, 172-176 (1988).

4. S. Jutamulia and T. Asakura, "Rotation-invariant joint transform correlator," Appl. Opt. 33, 5440-5442 (1994).

5. R. D. Juday, "Optimal realizable filters and the minimum Euclidean distance principle," Appl. Opt. 32, 5100-5111 (1993).

6. J. L. Horner and P. D. Gianino, "Applying the phase-only filter concept to the synthetic discriminant function correlation filter," Appl. Opt. 24, 851-855 (1985). 
7. D. Casasent and W. A. Rozzi, "Computer-generated and phaseonly synthetic discriminant function filters," Appl. Opt. 25, 3767-3772 (1986).

8. R. R. Kallman, "Direct construction of phase-only filters," Appl. Opt. 26, 5200-5201 (1987).

9. D. A. Jared and D. J. Ennis, "Inclusion of filter modulation in synthetic-discriminant-function construction," Appl. Opt. 28, 232-239 (1989).

10. M. Montes-Usategui, J. Campos, and I. Juvells, "Computation of arbitrarily constrained synthetic discriminant functions," Appl. Opt. 34, 3904-3914 (1995).

11. R. D. Juday, "Generality of matched filtering and minimum Euclidean distance projection for optical pattern recognition," J. Opt. Soc. Am. A 18, 1882-1896 (2001).

12. M. B. Reid, P. W. Ma, J. D. Downie, and E. Ochoa, "Experimental verification of modified synthetic discriminant function filters for rotation invariance," Appl. Opt. 29, 1209-1212 (1990).
13. P. C. Miller and R. S. Caprari, "Demonstration of improved automatic target-recognition performance by moment analysis of correlation peaks," Appl. Opt. 38, 1325-1331 (1999).

14. D. W. Carlson and B. V. K. Vijaya Kumar, "Synthetic discriminant functions for implementations on arbitrarily constrained devices," in Optical Information Processing Systems and Architectures, B. Javidi, ed., Proc. SPIE 1772, 10-20 (1992).

15. C. Zeile and E. Lüder, "Complex transmission of liquid crystal light modulators in optical signal processing applications," in Liquid Crystal Materials, Devices, and Applications II, U. Efron and M. D. Wand, eds., Proc. SPIE 1911, 195-206 (1993).

16. E. Martín-Badosa, A. Carnicer, I. Juvells, and S. Vallmitjana, "Complex modulation characterization of liquid crystal devices by interferometric data correlation," Meas. Sci. Technol. 8, 764-772 (1997).

17. M. Montes-Usategui, S. E. Monroe, and R. D. Juday, "Automated self-alignment procedure for optical correlators," Opt. Eng. 36, 1782-1791 (1997). 